\title{
Caries Experience among Females aged 16-21 in Punjab, India and its Relationship with Lifestyle and Salivary HSP70 Levels
}

\author{
Rabinder Kaur ${ }^{a}$ \\ Hardeep Kataria ${ }^{b}$ \\ Sushil Kumarb \\ Gurcharan Kaur
}

\begin{abstract}
Objectives: The objective of the current study was to assess the prevalence and means of dental caries experience and the prevalence of calculus among college students and their awareness of general dental health. The correlation between the experience of caries and extracellular levels of heat shock protein-70 (eHSP70) was studied in saliva samples of these students to ascertain whether eHSP level changes as a result of increased caries experience of these subjects.

Methods: The current study was conducted among 147 female undergraduate students aged 1621 in the state of Punjab, India. Dental examinations were performed to assess for the presence of calculus and DMFT, and subjects were asked to fill in a questionnaire. The data were recorded and analysed for the role of various factors in the prevalence of caries, including dietary habits. Saliva samples were collected for ELISA-based assay of eHSP70 levels.

Results: The prevalence of caries experience (DMFT>0) was 63.2\%, with average DMFT of 2.91 in students aged $16-18$ and 3.26 in those aged $19-21$. Of those with caries, $68 \%$ also showed the presence of calculus, compared with $60.0 \%$ of subjects with DMFT $=0$. The level of eHSP was significantly higher $(P<.05)$ in subjects with higher levels of caries (DMFT=4-8) compared with subjects having DMFT between 0 and 3 .

Conclusions: A significant relationship was observed between caries experience and oral hygiene habits, emphasizing the need to design and plan preventive strategies for persons at greatest risk. The correlation between mean DMFT and prevalence of eHSP was highly significant. This is the first study to report a correlation between caries experience and the saliva level of stress response protein HSP70. (Eur J Dent 2010;4:308-313)
\end{abstract}

Key words: Dental caries; HSPs; Dental health; DMFT; Calculus.

a Baba Jaswant Singh Dental College Hospital and Research Institute, Punjab, India.

b Department of Biotechnology, Guru Nanak Dev University, Punjab, India.

- Corresponding author: Professor Gurcharan Kaur Department of Biotechnology, Guru Nanak Dev University, Amritsar-143005, Punjab, India.

Phone: 091-183-2451532

Fax: 091-183-2258820

E-mail: kgurcharan.neurodyahoo.com

\section{INTRODUCTION}

Dental caries and related oral diseases like gingivitis and periodontitis are among the most common dental diseases, with a very high prevalence in both developed and developing countries, affecting people from all walks of life. ${ }^{1}$ The prevalence of these diseases is constantly increasing with changes in dietary habits and increased 
consumption of fast food by the younger generation. The prevalence of dental caries in children and adults is approximately $60-65 \%$ in India. ${ }^{2-4}$ The available epidemiological data clearly reflect a marked increase in the prevalence of dental caries in many developed and developing countries. ${ }^{1}$ Significantly high percentages of untreated caries at the level of cavity have been reported across all age groups and among all socio-demographic characteristics. 5

Heat shock proteins (HSPs) are expressed in low concentration in a basal state, but exposure of cells to glucose analogues, heavy metals, ischemia, sodium arsenite, endotoxemia, free radical formation, microbial infections, nitric oxide, hormones, and antibiotics also induce the expression of HSPs. ${ }^{711}$ An accumulation of evidence has shown that environmental and pathological stresses induce HSPs, especially the inducible form of HSP70 and HSP72. The degree of induction depends on the level and duration of exposure to stress. ${ }^{12}$ Some evidence has been published showing that chaperons are also present outside the cell, exerting cytokine effects and immune recognition. HSP70 is present in human sera and saliva as well. ${ }^{13}$

The aim of this study was to describe the prevalence and means of caries experience and the prevalence of calculus among female students attending various colleges in Punjab, India, and their relationship to lifestyle. Further, the correlation between caries experience and extracellular levels of heat shock protein-70 (eHSP70) was studied in saliva samples of these students.

\section{MATERIALS AND METHODS}

The present study comprised 147 subjects. All these cases were selected from female students aged 16-21 and attending undergraduate classes at local colleges in Punjab, India. Students were randomly selected from a list of candidates in UG classes, and every individual had an equal chance of selection. A simple probability random sampling method was used in obtaining information about caries experience (DMFT) and calculus and their relationship to eHSP levels in saliva samples. The researchers adhered to WHO guidelines while preparing the questionnaire for data collection. Ethical approval of the study was obtained from college authorities, and written consent was obtained from the students. Oral examination of all subjects was performed by a trained dentist (RK) who examined all exposed and accessible surfaces for caries. The students were examined while seated in an ordinary chair, in broad daylight and facing away from direct sunlight. The dental examination for carious teeth involved sequential assessment of teeth, from 1 to 32 . The dental examination was performed with the help of illumination devices, and data were recorded for analysis. A mirror and explorer were also used during the examination. Compressed air was used to dry teeth and to remove debris to allow better visual examination. The instruments used were sterilized after every single use. The site presenting dental caries (decayed teeth, DT) at a cavity level was recorded, and filled (filled teeth, FT) and missing teeth (MT) were counted separately; data are presented as DMFT, DT, FT, and MT. Subjects were asked about the level of caries and calculus as well as the duration of symptoms in a questionnaire. The questionnaire, given to the sample group, also investigated oral hygiene, lifestyle, and snack habits of the subjects.

\section{ELISA for eHSP assay}

The primary antibodies used for ELISA were monoclonal anti-HSP70 (clone-BRM-22, SIGMA), which recognizes both constitutive and inducible forms of HSP70. The secondary antibody used was anti-mouse IgG, peroxidase linked antibody (Bangalore Geneil. All other chemicals and reagents were the purest available commercially from local suppliers.

\section{Saliva sample collection}

Saliva was collected during the forenoon between 10.00 and 12.00. Participants were told to first use mouthwash and then allowed to chew on a cotton ball. When stimulated saliva accumulated in their mouth for 4-5 min, it was transferred to a collecting vessel. Saliva samples were precleared by centrifugation (15,000 rpm, $4^{\circ} \mathrm{C}$ for $\left.15 \mathrm{~min}\right)$, and sterile filtered with 0.22 pore size filters and stored at $-20^{\circ} \mathrm{C}$ for further use. Saliva HSP level was determined by indirect ELISA. Saliva was diluted to a 1:1 ratio with a coating buffer $\left(\mathrm{Na}_{2} \mathrm{CO}_{3}-\right.$ $\mathrm{NaHCO}_{3}$ buffer $0.05 \mathrm{M}$, 9.6). From this diluted sample, $100 \mu \mathrm{l}$ of sample (in triplicate) was used to coat each well of a microassay plate. The plate 
was covered and incubated overnight at $4^{\circ} \mathrm{C}$ for the antigen coating. After incubation, liquid was aspirated from all wells, and wells were washed with wash buffer (PBST 0.1\%) 3-4 times. Then $100 \mu \mathrm{l}$ of blocking solution ( $1 \%$ BSA) was added to each well and the plate was incubated for $2 \mathrm{~h}$ at $37^{\circ} \mathrm{C}$. After blocking, $100 \mu \mathrm{l}$ of primary antibody anti-HSP70 11:500 diluted) was added to each well and the plate was incubated for $2 \mathrm{~h}$ at $37^{\circ} \mathrm{C}$. Again, after incubation, the liquid was removed and wells were washed with wash buffer 3-4 times. Following washings, secondary anti-mouse IgG, peroxidase linked antibody (1:500 diluted antibody) was added to each well, and the plate was again incubated at $37^{\circ} \mathrm{C}$ for $2 \mathrm{~h}$. After 3 washings, $100 \mu \mathrm{l}$ of OPD (Ortho Phenyl Diamine) substrate solution was added to each well and the assay plate was incubated for 30 min in darkness, then $50 \mu \mathrm{l}$ of acid stop solution was added to terminate the reaction. Absorbance was read at $492 \mathrm{~nm}$ using a multiscan ELISA absorbance plate reader, and optical density values were compared between no, low, and high groups of caries experience subjects.

\section{Statistical analysis}

Means and standard deviations were calculated to express DMFT, DT, MT, and FT values as well as eHSP level in no, low, and high caries experience groups. Student's t test was applied to compare differences in prevalence of dental caries among age and eHSP levels of the different groups.

\section{RESULTS}

The prevalence of dental caries experience and mean DMFT among these college students were analyzed in two age groups (Table 1). Both the prevalence of caries experience $(58.80 \%)$ as well as mean DMFT $(3.26 \pm 0.31)$ were not significantly different in the upper age group (19-21 years) compared with the group aged 16-18 167.60\%, $2.91 \pm 0.21$ ). Of the total 93 students with caries experience, only 28 (about $30 \%$ ) showed 1-3 fillings,

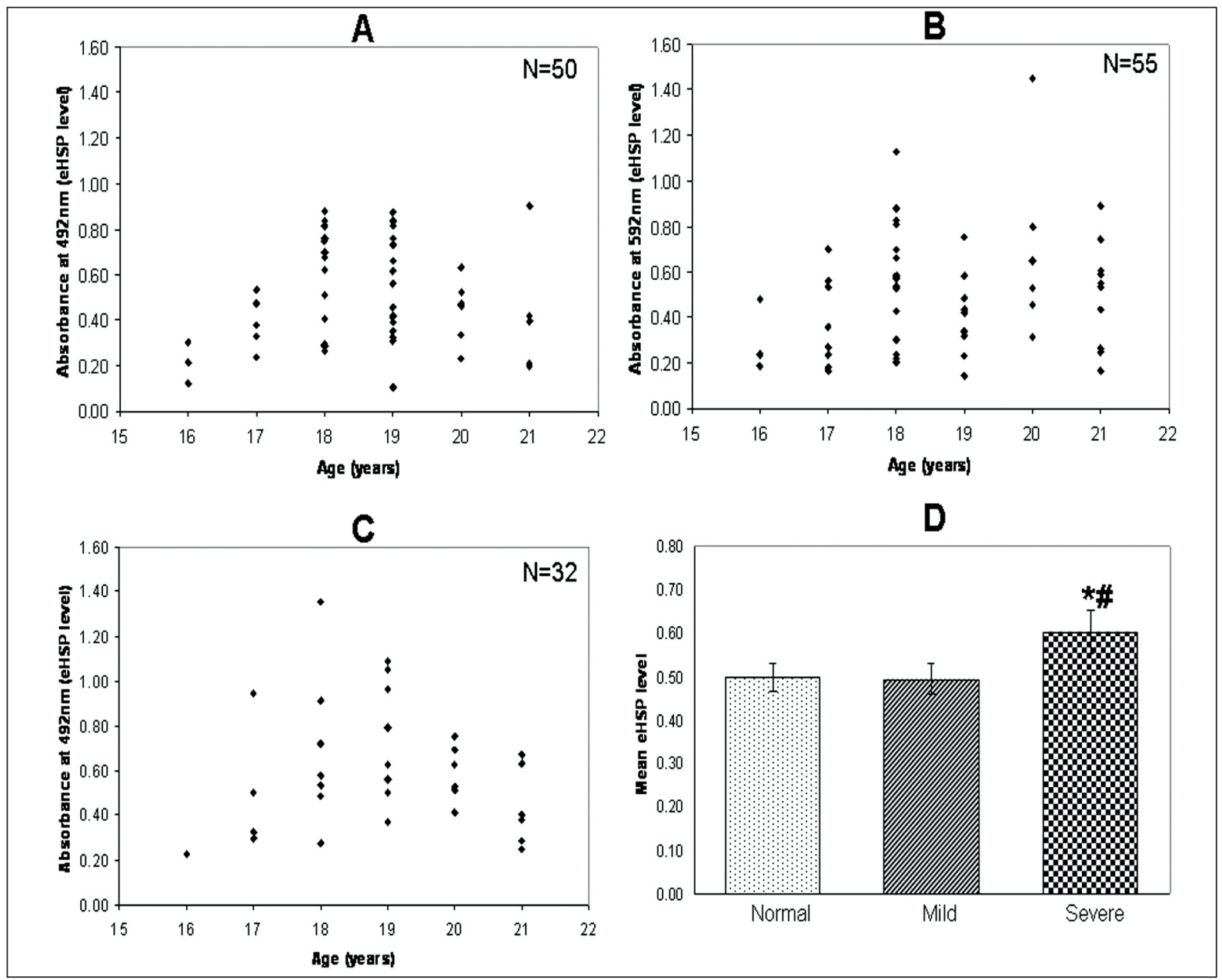

Figure 1. Distribution of salivary HSP in (A) normal subjects with DMFT=0, (B) mild dental caries with DMFT=1-3 and (C) severe dental caries with DMFT=4-8 of subjects in the age group of 16-21 years. The mean value (Mean \pm S.E.M) of eHSP in normal, mild and severe subjects is represented as histogram in (D). Statistically significant difference was found between Normal and Severe $\left.{ }^{*}\right)$ as well as Mild and Severe (\#) groups $(\mathrm{P}<.05)$. 
and $9(10 \%)$ showed 1-2 missing teeth along with filled teeth, whereas the majority of subjects (70\%) showed decayed teeth with no fillings or missing teeth attributed to caries.

Besides caries experience, these students were examined for the presence of calculus. Approximately $60 \%$ of subjects with no caries experience and $67 \%$ of subjects with caries experience showed the presence of calculus (Table 2). Preventive care such as scaling, fluoride-supplemented toothpaste, and diet counseling was required in 123 (84\%) subjects. These data were retrieved from the information collected in the questionnaire. Oral hygiene and lifestyle factors such as dietary and exercise habits were also analyzed in these students. All students reported brushing their teeth 1-2 times daily, but only $20 \%$ of subjects were aware of the fluoride supplement in their toothpaste. A significant association between caries experience, calculus, and frequency of tooth brushing was evident from the data collected in the questionnaire. Daily eating of junk food, chocolate, candy, soft drinks, chips, etc. was reported by $75 \%$ of subjects, and only $52 \%$ reported engaging in some daily physical activity such as yoga, dance, or walking.

Correlation between DMFT and eHSP level was assessed, and the results are shown in Figure 1. The mean eHSP level was significantly higher in the group showing 4-8 decayed teeth (DMFT=4-8; 0.602 [average optical density]) compared with subjects without dental caries (DMFT=0; 0.499) and those with 1-3 decayed teeth (DMFT=1-3; 0.494). Results are shown as scatter plots in Figure $1 \mathrm{~A}, \mathrm{~B}$, and $\mathrm{C}$, and mean \pm SEM values are shown in Figure 1D with statistical analysis. The

Table 1. Percent infection with dental caries in different age groups and average DMFT.

\begin{tabular}{|c|c|c|c|c|c|}
\hline \multirow[b]{2}{*}{ Age group } & \multicolumn{2}{|c|}{ Normal } & \multicolumn{2}{|c|}{ Dental caries } & \multirow{2}{*}{$\begin{array}{c}\text { DMFT } \\
\text { Mean } \pm S D\end{array}$} \\
\hline & No. & $\%$ & No. & $\%$ & \\
\hline $16-18$ & 22 & 32.3 & 46 & 67.6 & $2.91 \pm 1.72$ \\
\hline $19-21$ & 33 & 41.2 & 47 & 58.8 & $3.26 \pm 2.10$ \\
\hline
\end{tabular}

mean value for eHSP of the entire study group was 0.521 . On interanalysis of the percentage of subjects showing eHSP level less and greater than mean value, it was observed that $58 \%$ of subjects with DMFT $=0$ showed eHSP level lower than mean value, whereas this percentage was reversed in subjects showing DMFT=4-8 (Table 3).

\section{DISCUSSION}

The purpose of the study was to provide data on oral health status and its correlation with social-behavioral factors and the level of eHSPs in a sample of undergraduate female students aged 16-21. The study aimed to assess the prevalence of caries experience in this group and to make subjects aware of the need for future preventive interventions. Caries experience showed a level of disease comparable to that of other states in India as well as of many developed and developing countries. Overall caries prevalence was found to be $63.2 \%$ in the current study. Although not statistically significant, average DMFT was higher in the upper age group, i.e., 19-21 years. Previous studies have shown that $49 \%$ of 14 -year-old children showed evidence of caries experience in England and Wales; 13-18-year-olds in Milan, Italy, showed prevalence of $59.11 \%$, whereas in Uganda, $80 \%$ of students in the age group 13-19 years were positive for caries experience. ${ }^{14-16}$ Recently, a CDC surveillance summary of oral health in the U.S. population also reported that $49.6 \%$ of adolescents in the age group 12-15 showed evidence of caries experience. ${ }^{17}$ In the current study, the average DMFT was 3.09 for all subjects. Similar observations have been made in previous studies in rural and urban India. ${ }^{18-22}$ Previous studies con-

Table 2. Prevalence of calculus in subjects with and without dental caries

\begin{tabular}{|c|c|c|c|c|}
\hline Age group & $\begin{array}{c}\text { Normal } \\
\text { (Calculus) }\end{array}$ & $\%$ & $\begin{array}{c}\text { Dental caries } \\
\text { (Calculus) }\end{array}$ & $\%$ \\
\hline Number & 55 (33) & 60.0 & $93(60)$ & 66.7 \\
\hline
\end{tabular}

Table 3. Percentage of subjects showing eHSP level lower and higher than average value (0.521).

\begin{tabular}{lccc}
\hline & Normal & Mild & Severe \\
\hline eHSP lower than average & DMFT $=0$ & DMFT=1-3 & DMFT=4-8 \\
\hline eHSP higher than average & $29 / 50(58.0 \%)$ & $28 / 55(49.1 \%)$ & $14 / 32(43.7 \%)$ \\
\hline
\end{tabular}


ducted in Northern India in rural Punjab and rural Karnataka also reported an increase in mean DMFT scores with increasing age. 18,23,24 $^{2}$

The intra-analysis of DMFT in these subjects revealed that about $70 \%$ comprised decayed teeth, whereas missing and filled components were only about $30 \%$. The majority of subjects showed decayed teeth with no fillings or missing teeth as the result of caries, thereby indicating lack of awareness about and motivation for good oral health among the younger generation or barriers to access to dental services. The current results also emphasize the need to spread awareness among college students about oral health.

These subjects were also examined for knowledge about, attitude toward, and good practice of oral health (not described here in materials and methods) through a questionnaire designed for this purpose. The high caries experience and calculus prevalence seen in the current study of college students may be attributed to factors such as poor dietary and oral hygiene habits. Although many of the students reported brushing their teeth two times daily, a significant association between caries experience, calculus, and frequency of tooth brushing was evident (not described here in results). The majority of these students reported consuming sugary foods such as chocolate, candy, jellies, soft drinks, etc. approximately 3-5 times daily, which may be considered one of the most important factors in high caries experience in this group. Moreover, only $70 \%$ of subjects had knowledge of brushing frequency of three times per day, and only $30 \%$ practiced this habit of brushing three times daily with fluoridated toothpaste. The majority of subjects free of caries (DMFT=0) reported brushing their teeth twice or more daily, and they had healthy dietary and lifestyle habits, highlighting the role of maintenance of oral hygiene and good lifestyle habits in prevention of caries.

The present results showed a comparatively higher level of salivary HSP70 in subjects with $\mathrm{DMFT}=4$ or more compared with $\mathrm{DMFT}=0$ and $\mathrm{DMFT}=1-3$. The average eHSP level for all subjects was 0.521 (optical density measurement), and on inter-analysis of eHSP levels of three groups with $\mathrm{DMFT}=0, \mathrm{DMFT}=1-3$, and $\mathrm{DMFT}=4-8$, it was observed that more subjects showed above-average values for eHSP among those with DMFT=48 compared with subjects with $\mathrm{DMFT}=0$ and
$\mathrm{DMFT}=1-3$. Although changes in salivary HSP70 under stress conditions, especially bacterial infection in the oral cavity, have not been studied in detail, the current results showing higher salivary HSP70 in subjects with higher DMFT may be related to increased stress due to higher bacterial load in the oral cavity in subjects with dental caries. Recently, Fabian et al ${ }^{13,25,26}$ reported that salivary HSP70 levels change in response to psychological stressors and suggested that salivary HSP70 may have some immune-modulatory role in the oral cavity. These authors further suggested that salivary HSP70 may be a mixture of several known HSP70-type stress proteins, including HSP72 and HSP73. HSPs are important in physiological and pathological processes and also serve as biomarkers to evaluate the extent of disease or the degree of environmental stress. The current results showing a correlation between DMFT and salivary HSP70 levels further suggest a potential impact of salivary HSP70 on oral defense mechanisms under physiological and pathological stress conditions. Extracellular HSP70 is present in both healthy and diseased subjects and seems to play a dual role in the body: in healthy subjects, it plays an important role in immune regulation as well as in cytoprotection, whereas in the case of disease, extracellular HSP70 acts as a danger signal to immune cells, promotes the immune response, and improves host defense. ${ }^{28-30}$

\section{CONCLUSIONS}

Based on the present data and on previous studies, it can be concluded that the increasing prevalence of dental caries among the younger generation is predisposed by lifestyle and poor oral hygiene. The potential impact of salivary HSP70 on caries experience needs to be explored further.

\section{REFERENCES}

1. Bagramian RA, Garcia-Godoy F, Volpe AR. The global increase in dental caries. A pending public health crisis. Am J Dent 2009;22:3-8.

2. Shouri KL. Dental caries in Indian children. Indian J Med Res 1941;29:709-722.

3. Ramachandran K, Rajan BP, Shanmungan S. Epidemiological studies of dental disorders in Tamilnadu population, prevalence of dental caries and periodontal diseases. $J$ Indian Dent Assoc 1973;45:65-70. 
4. Dhar V, Jain A, Van Dyke TE, Kohli A. Prevalence of dental caries and treatment needs in the school-going children of rural areas in Udaipur district. J Indian Soc Pedod Prev Dent 2007;25:119-121.

5. Dye BA, Tan S, Smith V, Lewis BG, Barker LK, ThorntonEvans G, Eke PI, Beltrán-Aguilar ED, Horowitz AM, Li CH. Trends in oral health status: United States, 1988-1994 and 1999-2004. Vital Health Stat 11 2007; 248:1-92.

6. Milciuviene S, Bendoraitiene E, Andruskeviciene V, et al. Dental caries prevalence among 12-15-year-olds in Lithuania between 1983 and 2005. Medicina (Kaunas) 2009;45:6876.

7. Pouyssegur J, Shiu PC, Pastan I. Induction of two transformation-sensitive membrane polypeptides in normal fibroblasts by a block in glycoprotein synthesis or glucose deprivation. Cell 1977;11:941-947.

8. Johnston D, Oppermann H, Jackson J, Levinson W. Induction of four proteins in chick embryo cells by sodium arsenite. J Biol Chem 1980;255:6975-6980.

9. Levinson W, Oppermann $\mathrm{H}$ and Jackson J. Transition series metals and sulfhydryl reagents induce the synthesis of four proteins in eukaryotic cells. Biochim Biophys Acta 1980;606:170-180.

10. Adrie C, Richter C, Bachelet $M$, et al. Contrasting effects of $\mathrm{NO}$ and peroxynitrites on hsp70 expression and apoptosis in human monocytes. Am J Physiol Cell Physiol 2000;279:C452-460.

11. Ofenstein JP, Heidemann S, Juett-Wilstermann A, Sarnaik A. Expression of stress proteins HSP 72 \& HSP 32 in response to endotoxemia. Ann Clin Lab Sci 2000;30:92-98.

12. Kianga JG, Tsokos GC. Heat shock protein 70 kda: Molecular biology, biochemistry, and physiology. Pharmacol Ther 1998;80:183-201.

13. Fabian TK, Gaspar J, Fejerdy L, et al. Hsp70 is present in human saliva. Med Sci Monit 2003;9:BR62-65.

14. Pitts NB, Boyles J, Nugent ZJ, Thomas N, Pine CM. The dental caries experience of 14-year-old children in England and Wales. Surveys co-ordinated by the British Association for the Study of Community Dentistry in 2002/2003. Community Dent Health 2004;21:45-57.

15. Campus G, Cagetti MG, Senna A, Sacco G, Strohmenger L, Petersen PE. Caries prevalence and need for dental care in 13-18-year-olds in the municipality of Milan, Italy. Community Dent Health 2008;25:237-242.

16. Seibert W, Farmer-Dixon C, Bolden TE, Stewart JH. Assessing dental caries prevalence in African-American youth and adults. J Tenn Dent Assoc 2004;84:24-27.

17. Beltrán-Aguilar ED, Barker LK, Canto MT, Dye BA, Gooch BF, Griffin SO, Hyman J, Jaramillo F, Kingman A, NowjackRaymer R, Selwitz RH, Wu T. Centers for Disease Control and Prevention (CDC). Surveillance for dental caries, dental sealants, tooth retention, edentulism, and enamel fluorosis-United States, 1988-1994 and 1999-2002. MMWR Surveill Summ 2005;54:1-43.
18. Khera N, Tewari A, Chawla HS. Inter-comparison of prevalence and severity of dental caries in urban and rural areas of northern India. J Indian Soc Pedod Prev Dent 1984:2:1925.

19. Saha S, Sarkar S. Prevalence and severity of dental caries and oral hygiene status in rural and urban areas of Calcutta. J Indian Soc Pedod Prev Dent 1996:14:17-20.

20. Rao A, Sequeira SP, Peter S. Prevalence of dental caries among school children of Moodbidri. J Indian Soc Pedod Prev Dent 1999:17:45-48.

21. Goyal A, Gauba K, Chawla H, Kaur M, Kapur A. Epidemiology of dental caries in Chandigarh school children and trends over the last 25 years. In: Medknow Publications, 2007.

22. Khan AA, Jain SK, Shrivastav A. Prevalence of dental caries among the population of Gwalior (India) in relation of different associated factors. Eur J Dent 2008;2:81-85.

23. Gauba K, Tewari A, Chawla HS. Frequency distribution of children according to dental caries status in rural areas of northern India (Punjab). J Indian Dent Assoc 1986;58:505512.

24. Saravanan S, Kalyani V, Vijayarani MP, et al. Caries prevalence and treatment needs of rural school children in Chidambaram Taluk, Tamilnadu, south India. Indian J Dent Res 2008;19:186-190.

25. Fábián TK, Fejérdy $P$, Nguyen $M$, Sőti $C$, Csermely $P$. Potential immunological functions of salivary hsp70 in mucosal and periodontal defense mechanisms. Archivum Immunologiae et Therapiae Experimentalis 2007;55:91-98.

26. Fabian TK, Toth Z, Fejerdy L, Kaan B, Csermely P, Fejerdy $P$. Photo-acoustic stimulation increases the amount of 70 kda heat shock protein (HSP70) in human whole saliva. A pilot study. Int J Psychophysiol 2004;52:211-216.

27. Oeheler R, Pusch E, Zellner M, Dungel P, Hergovics N, Homonick M, Liasen MM, Brabec M, Roth E. Cell type-specific variations in the induction of hsp-70 in human leucocytes by fever like whole body hyperthermia. Cell Stress and Chaperons 2001;6:306-315.

28. Nardai G, Vegh EM, Prohaszka Z, Csermely P. Chaperonerelated immune dysfunction: An emergent property of distorted chaperone networks. Trends Immunol 2006:27:74-79.

29. Mellati AA. The role of heat shock proteins as chaperones on several human diseases. Saudi Med J 2006:27:13021305.

30. Malaitsev VV, Bogdanova IM, Makarova OV. Heat shock proteins and their role in the development of pathological processes. Arkh Patol 2008:70:31-38. 